Artículo

\title{
Variación porcentual del INPC, canasta alimentaria y salario mínimo en México, enero 2002-junio 2020
}

\author{
Julio César Ayllon Benítez ${ }^{1 \S}$ \\ José Miguel Omaña Silvestre ${ }^{1}$ \\ Dora Ma. Sangerman-Jarquín ${ }^{2}$ \\ Miguel Ángel Martínez Damián ${ }^{1}$ \\ Jaime Arturo Matus Gardea ${ }^{1}$ \\ Felipe de Jesús González Razo ${ }^{3}$
}

${ }^{1}$ Colegio de Postgraduados. Carretera México-Texcoco km 36.5, Montecillo, Texcoco, Estado de México. CP. 56230. Tel. 595 9520200, ext. 1839. ${ }^{2}$ Campo Experimental Valle de México-INIFAP. Carretera Los Reyes-Texcoco km 13.5, Coatlinchán, Texcoco, Estado de México. CP. 56250. (sangerman.dora@inifap.gob.mx). ${ }^{3}$ Centro Universitario Temascaltepec-Universidad Autónoma del Estado de México. Carretera Toluca-Cd. Altamirano km 67.5, Col. Barrio de Santiago s/n, Temascaltepec, Estado de México. CP. 51300.

${ }^{\S}$ Autor para correspondencia: julio.ayllon@colpos.mx.

\section{Resumen}

En México, el Consejo Nacional de Evaluación de la Política de Desarrollo Social utiliza la denominación de pobreza por ingreso, para tal fin emplea la línea de bienestar mínimo, el cual se refiere a las personas cuyos ingresos son insuficientes para adquirir la canasta básica recomendable, equivalente al valor de la canasta alimentaria por persona y el de línea de bienestar, semejante a la anterior denominación de pobreza patrimonial, equivalente al valor de la canasta alimentaria y no alimentaria por persona al mes. Constitucionalmente el salario mínimo debe cubrir las necesidades de una familia, en orden material, social y cultural y para proveer educación. El periodo de estudio fue de enero 2002 a junio 2020. La información se procesó en el programa estadístico SAS ${ }^{\circledR}$. El objetivo del presente trabajo fue estimar la tasa de variación porcentual del índice nacional de precios al consumidor, precios de la canasta alimentaria rural y urbana y salario mínimo general para explicar el diferencial existente entre este último y comprobar su efecto al estar sobre o debajo de la variación porcentual del índice nacional de precios al consumidor y VPPCA. En episodios de alta inflación, principalmente en el aspecto alimentario, periodos 2004, 2007-2009, 2012-2013 y 2017 el SMG apenas ha compensado parte del aumento, acentuándose el panorama en 2017; sin embargo, aumentos importantes ocurridos desde 2015 y en los últimos periodos (2019 y 2020) en el SMG nominal en 16 y $20 \%$ han sido importantes para reducir la brecha entre el SMG real.

Palabras clave: economía, precios, salario mínimo, vulnerabilidad.

Recibido: mayo de 2021

Aceptado: junio de 2021 


\section{Introducción}

El índice nacional de precios al consumidor (INPC) es un indicador económico que facilita la toma de decisiones económicas inherentes al comportamiento de los precios, ello se debe a que brinda información al gobierno, las empresas y las familias sobre los cambios que tiene el costo de la vida en el país (Flores, 2017), al seguir el comportamiento de los precios al por menor de una canasta de bienes y servicios, donde su incremento o variación porcentual (VP) corresponde a la medida más empleada para la inflación (Alonso y Rivera, 2017). En la metodología del Instituto Nacional de Estadística y Geografía (INEGI), para que el año base de referencia fuera segunda quincena de julio 2018 $=100$, cotizaron 55 ciudades (ubicadas en las 32 entidades del país), para recabar en promedio 159500 precios quincenalmente, agrupados en 299 conceptos de consumo genéricos, los cuales abarcan 91 ramas de actividad económica.

Las cotizaciones se procesan, para dar lugar a índices de precios, de los cuales se desprenden los índices nacionales oficiales que contemplan conceptos de consumo familiar de acuerdo a cierta canasta de bienes y servicios que consideró el total del gasto en consumo de los hogares, ya que la estructura de ponderación (actualizados a la segunda quincena julio 2018, vía precios relativos [el gasto de cada genérico se actualiza de acuerdo a la variación de sus respectivos índices de precios]) se obtuvo de los gastos reportados en las Encuestas Nacionales de Gastos de los Hogares (ENIGH) 2012 y 2013 (encuesta levantada por dos años consecutivos en 64000 hogares, con una encuesta basada en un catálogo de gastos comparable internacionalmente), en complementación con la ENIGH 2014 (INEGI, 2018). Uno de los aspectos más importante es el componente del INPC no subyacente (excluye precios de alimentos y energía), el cual las autoridades gubernamentales se interesan en medir las tendencias, en este rubro entran los precios de algunos bienes muy volátiles, lo que indica que los cambios de precios a veces son temporales (Dornsbusch et al., 2009).

En el periodo de mayor inflación interanual observada hasta octubre 2017, la variación porcentual del INPC (VPINPC) se ubicó en 6.37\%, siendo superior en 3.31 puntos porcentuales con relación al mismo mes de referencia 2016 (3.06\%). Dentro del índice subyacente, los precios de las mercancías y los servicios tuvieron una variación interanual de 5.97 y $3.75 \%$ respectivamente, en contraparte el índice no subyacente (11.4\%) los productos agropecuarios reportaron una variación en los precios de $8.37 \%$ ubicándose en 3.12 puntos porcentuales arriba de la registrada el mismo periodo 2016 (5.25\%), respecto a los energéticos y tarifas autorizadas por el gobierno, mostraron aumento en sus precios de $13.36 \%$, siendo mayor 11.84 puntos porcentuales en relación al presentado en 2016 cuando fue de $1.52 \%$ (CEFP, 2017).

Mientras, el Consejo Nacional de Evaluación de la Política de Desarrollo Social (CONEVAL) menciona que el principal factor que incide en la identificación de la pobreza es precisamente la capacidad adquisitiva del ingreso familiar, justamente en materia alimentaria.

La medición de la pobreza en México realizada por el CONEVAL, utiliza la denominación de pobreza por ingreso, bajo un esquema metodológico induce y evalúa una canasta alimentaria de precios de productos en el ámbito rural y urbano; es decir, la medición de pobreza utiliza dos líneas de ingreso: línea de pobreza extrema por ingresos (LBM), que equivale al valor de la canasta alimentaria (CA) por persona al mes y línea de pobreza por ingresos (LB), que equivale al valor total de la canasta alimentaria y no alimentaria por persona al mes. En los últimos años 2014, 2016 
y 2018 la población con ingreso inferior a la LBM ha sido de 24.6, 21.4 y 21 millones de personas, cifras que representaron el $20.6,17.5$ y $16.8 \%$ de la población, mientras que el comportamiento en la LB ha sido de 63.8, 62 y 61.1 millones de personas, siendo 53.2, 50.6 y 48.8\% de la población (CONEVAL, 2020).

La construcción de la canasta alimentaria sirve de referencia para obtener el valor de la línea de pobreza extrema por ingresos, dentro de las generalidades de la metodología considera la selección del estrato poblacional de referencia (EPR) cuya aproximación de consumo coincide con alguna recomendación nutricional, se analiza el patrón de consumo y la lista de productos y cantidades que consume el estrato de acuerdo con los rubros contenidos en la ENIGH 2016; asimismo, clasifica los alimentos en 45 rubros y se obtiene un porcentaje de la frecuencia de consumo y del gasto en alimentos, seleccionando aquellos productos donde su porcentaje de frecuencia de consumo de alimentos con respecto a su rubro sea mayor a 10 y que el porcentaje de gasto de cada alimento con respecto al total sea mayor de $0.5 \%$.

Para el valor de la CA se generó una base de precios implícitos a partir de la información de la ENIGH, donde el precio implícito de cada rubro se calculó como la media geométrica de las razones entre el gasto y la cantidad por rubro de todos los hogares para posteriormente generar el costo de la canasta al multiplicar el precio por el consumo de cada alimento y así obtener el costo mensual al sumar los costos de todos los alimentos que la componen, multiplicado por 30 (CONEVAL, 2018).

Este trabajo tuvo por objetivo analizar la diferencia salarial y la manera de relacionarse con el ritmo de los niveles de precios, siendo la VPINPC una de las variables más importantes que han marcado el crecimiento o descenso de los salarios, el cual es percibido por cualquier clase, donde se logra observar cómo en algunos episodios del tiempo tiene un posible efecto de pérdida de poder adquisitivo al estar situado por debajo de la inflación general y de los PCA, a lo largo de los años en México.

\section{Materiales y métodos}

Se consideró la tasa de variación o inflación porcentual (VP) del INPC (INEGI, 2020), PCA rural y urbana (CONEVAL, 2020) y del SMG (CONASAMI, 2020). De acuerdo con INEGI (2020) y Brambila (2011), para determinar la tasa de variación porcentual del periodo se utilizó la siguiente ecuación: $\mathrm{VP}=\left[\left(\frac{\mathrm{VF}-\mathrm{VI}}{\mathrm{VI}}\right)\right] * 100$. Donde: $\mathrm{VP}=$ variación porcentual (dando origen a las variables); VPINPC = variación porcentual del INPC; VPPCA= variación porcentual de los PCA rural y urbana; y VPSMG= variación porcentual del SMG (nominal y real); VF= valor final; $\mathrm{y}$ $\mathrm{VI}=$ valor inicial. Asimismo, el estudio se basó en Noriega (2019), el cual segmenta los periodos de inflación alimentaria, principalmente donde ocurren movimientos abruptos, que elevan los indicadores. Con la innovación de comparar los distintos índices respecto al ritmo de crecimiento de la VPSMG, para descubrir si verdaderamente la política de aumento salarial ha compensado parte del incremento de precios en México. La información y los datos fueron procesados en el programa estadístico SAS ${ }^{\circledR}$. 


\section{Resultados y discusión}

El Cuadro 1, muestra una descripción del INPC base 2018, PCA rural y urbana, y SMG promedio, asimismo, su VP de cada una en el periodo 2002-2020. En términos nominales, el Cuadro 1 muestra acontecimientos de alta inflación en los PCA como en 2004, 2007-2009, 2012 y 2017. A inicios de 2006 existía una marcada tendencia en el incremento de los precios que se extendió hasta 2009.

Cuadro 1. Valor y variación porcentual (VP) promedio del INPC, PCA y SMG nominal.

\begin{tabular}{|c|c|c|c|c|c|c|c|c|c|c|c|}
\hline \multicolumn{3}{|c|}{$\mathrm{INPC}^{1 /}$} & \multicolumn{2}{|c|}{ PCA rural } & \multirow{2}{*}{$\begin{array}{c}\text { Diferencia } \\
\text { VPPCA- } \\
\text { VPINPC } \\
(\%)\end{array}$} & \multicolumn{2}{|c|}{ PCA urbana } & \multirow{2}{*}{$\begin{array}{c}\text { Diferencia } \\
\text { VPPCA- } \\
\text { VPINPC } \\
(\%)\end{array}$} & \multicolumn{2}{|c|}{ SMG } & \multirow{2}{*}{$\begin{array}{c}\text { Diferencia } \\
\text { VPSMG- } \\
\text { VPINPC } \\
(\%)\end{array}$} \\
\hline Año & $\begin{array}{c}\text { INPC } \\
\text { (unidad) }\end{array}$ & $\begin{array}{c}\text { VPINPC } \\
(\%)\end{array}$ & $\begin{array}{c}\text { Valor } \\
\text { (\$/pers- } \\
\text { mes) }\end{array}$ & $\begin{array}{c}\text { VPPCA } \\
(\%)\end{array}$ & & $\begin{array}{c}\text { Valor } \\
\text { (\$/pers- } \\
\text { mes) }\end{array}$ & $\begin{array}{c}\text { VPPCA } \\
(\%)\end{array}$ & & $\begin{array}{c}\text { Valor } \\
\text { (\$/pers- } \\
\text { mes) }\end{array}$ & $\begin{array}{c}\text { VPSMG } \\
(\%)\end{array}$ & \\
\hline 2002 & 51.92 & 5.03 & 427.67 & 4.61 & -0.42 & 618.94 & 4.95 & -0.08 & 1192.2 & 5.78 & 0.75 \\
\hline 2003 & 54.28 & 4.55 & 451.26 & 5.53 & 0.98 & 649.98 & 5.02 & 0.47 & 1245.9 & 4.5 & -0.05 \\
\hline 2004 & 56.83 & 4.69 & 483.97 & 7.22 & 2.53 & 694.2 & 6.78 & 2.09 & 1298.9 & 4.25 & -0.44 \\
\hline 2005 & 59.09 & 3.99 & 509.93 & 5.54 & 1.55 & 732.72 & 5.65 & 1.66 & 1357.2 & 4.49 & 0.5 \\
\hline 2006 & 61.24 & 3.63 & 509.93 & 5.54 & 1.91 & 732.72 & 5.65 & 2.02 & 1411.5 & 4 & 0.37 \\
\hline 2007 & 63.67 & 3.97 & 572.66 & 6.96 & 2.99 & 814.98 & 6.41 & 2.44 & 1466.4 & 3.89 & -0.08 \\
\hline 2008 & 66.93 & 5.12 & 610.6 & 6.64 & 1.52 & 869.92 & 6.74 & 1.62 & 1525.2 & 4.01 & -1.11 \\
\hline 2009 & 70.48 & 5.3 & 673.63 & 10.38 & 5.08 & 952.09 & 9.48 & 4.18 & 1595.7 & 4.62 & -0.68 \\
\hline 2010 & 73.41 & 4.16 & 697.93 & 3.68 & -0.48 & 990.78 & 4.11 & -0.05 & 1673.1 & 4.85 & 0.69 \\
\hline 2011 & 75.91 & 3.41 & 723.15 & 3.63 & 0.22 & 1029.1 & 3.87 & 0.46 & 1741.8 & 4.11 & 0.7 \\
\hline 2012 & 79.03 & 4.11 & 790.6 & 9.34 & 5.23 & 1112.6 & 8.12 & 4.01 & 1815 & 4.2 & 0.09 \\
\hline 2013 & 82.04 & 3.81 & 838.22 & 6.09 & 2.28 & 1180.9 & 6.19 & 2.38 & 1893.6 & 4.33 & 0.52 \\
\hline 2014 & 85.33 & 4.02 & 873.49 & 4.21 & 0.19 & 1244.7 & 5.4 & 1.38 & 1967.4 & 3.9 & -0.12 \\
\hline 2015 & 87.65 & 2.72 & 908.8 & 4.06 & 1.34 & 1279.5 & 2.81 & 0.09 & 2049.9 & 4.19 & 1.47 \\
\hline 2016 & 90.13 & 2.82 & 955.06 & 5.1 & 2.28 & 1337.3 & 4.52 & 1.7 & 2191.3 & 6.9 & 4.08 \\
\hline 2017 & 95.57 & 6.04 & 1021.9 & 7.02 & 0.98 & 1433.9 & 7.23 & 1.19 & 2401.2 & 9.58 & 3.54 \\
\hline 2018 & 100.26 & 4.9 & 1064.7 & 4.26 & -0.64 & 1500.8 & 4.72 & -0.18 & 2650.8 & 10.39 & 5.49 \\
\hline 2019 & 103.9 & 3.64 & 1112.3 & 4.5 & 0.86 & 1566.3 & 4.38 & 0.74 & 3080.4 & 16.21 & 12.57 \\
\hline 2020 & 106.47 & 2.48 & 1243.6 & 4.85 & 2.37 & 1632.9 & 4.19 & 1.71 & 3696.6 & 20 & 17.52 \\
\hline
\end{tabular}

${ }^{1 /}$ Base 2018=100. Datos de INEGI (2020); CONEVAL (2020).

El equilibrio inflacionario se interrumpió al resentir aumentos de precios en cultivos básicos en el mercado mundial, sin embargo, en 2008 precios de insumos agrícolas y petróleo en el mundo descendieron, mientras la inflación mantuvo elevadas tasas de crecimiento (CEDRSSA, 2017). En la diferencia obtenida de la variación porcentual o inflación de precios al consumidor y la inflación de precios de la canasta alimentaria donde se puede notar como en marcados años de inflación de la canasta alimentaria, esta sobrepasaba a veces hasta cuatro puntos, respecto a la inflación general, siendo el año más notorio 2009 y 2012. 
Al analizar el periodo 2007-2009 la VPCA rural y urbana en el primer año rondo más de seis puntos porcentuales, siendo superior a la inflación general (3.97\%) casi al doble a esta última. En 2009 la inflación general fue de 5.31\% mientras que el porcentaje de variación en la canasta alimentaria rural y urbana era de $10.38 \%$ y $9.48 \%$ en promedio, comportándose por arriba de la inflación general $(5.31 \%)$, con aumentos exiguos en el SMG de $4.62 \%$.

Una mejoría se notó en 2010 y 2011 en la VPPCA rural y urbana al lograr estar en 3.68 y $4.11 \%$ en el primer año, mientras en el segundo año 3.63 y 3.87\% respectivamente, al igual la VPSMG debido a que reportó incrementos de 4.85 y $4.11 \%$ para el periodo referenciado. En 2015 la VPCA rural continúo comportándose como años anteriores $(4.06 \%)$, caso contrario la VPCA urbana con significativas mejorías $(2.81 \%)$, año que mantuvo el nivel más bajo la inflación general $(2.72 \%)$.

Ante tal panorama en 2015 la VPSMG lograba estar en 4.19\%, precisamente en este año apenas y el salario ajustó a los aumentos del INPC y PCA para adquirir dicha cesta, sin embargo, los PCA crecían más por arriba del SMG. Sin embargo, en 2017 fue un año donde la inflación general presentó el nivel más alto en $6.04 \%$, situándose por arriba del objetivo de inflación (3\%) de BANXICO, impacto que se vio reflejado en la CA rural y urbana (7.02 y $7.23 \%)$ respectivamente. Ante tal escenario y debido a que el valor de una CBA es por persona y por mes, de acuerdo con CONEVAL en su concepto de pobreza extrema se refiere a una persona que muestra insuficiencia para obtener dicha canasta, a pesar de que haga uso de todo su ingreso en comprarla, de ser así, asumiendo que es jefe de familia, la población presentó dificultad para satisfacer sus necesidades básicas y las de su familia precariamente.

Para quien percibe un SMG, de 1999 a 2014 fue insuficiente, apenas cubría la adquisición de la CA a partir de 2015 el poder adquisitivo del SMG comenzó a mejorar en términos de esta canasta y en 2019 con el incremento de \$88.36 a \$102.68 pesos diarios se logró alcanzar dicha canasta al $100 \%$, acción que consiguió que una persona que lo percibe superará la LBM (CONASAMI, 2020).

La Figura 1, muestra una comparación de las distintas VP o inflación desde enero 2002 hasta junio 2020 (2002:01-2020:06), donde se observó que antes de 2015 la VPSMG nominal se comportaba en niveles inferiores a la inflación de precios de la CA rural y urbana. Los niveles de precios han sido superiores al ritmo de crecimiento del SMG; sin embargo, los mejores años han sido 2010, 2011 y 2015; asimismo, en este último es cuando empieza a ubicarse la tasa de variación porcentual del SMG nominal por encima de los niveles de precios.

Sin embargo, el deterioro que tuvo la capacidad adquisitiva del SMG en las últimas cuatro décadas, principalmente durante los ochenta y noventa del siglo XX, fue tan elevado (cercano a $80 \%$ ), que la recuperación del SMG real en los últimos años termina siendo marginal (Díaz, 2020). Tasas de inflación altas en 2017 y acontecimientos ocurridos en este mismo año como la contracción del subsidio gubernamental a gasolinas e iniciar la liberación de precios, aumento este indicador, acompañado de alta volatilidad en el tipo de cambio, repercutiendo en el ámbito alimentario (CEDRSSA, 2017). 


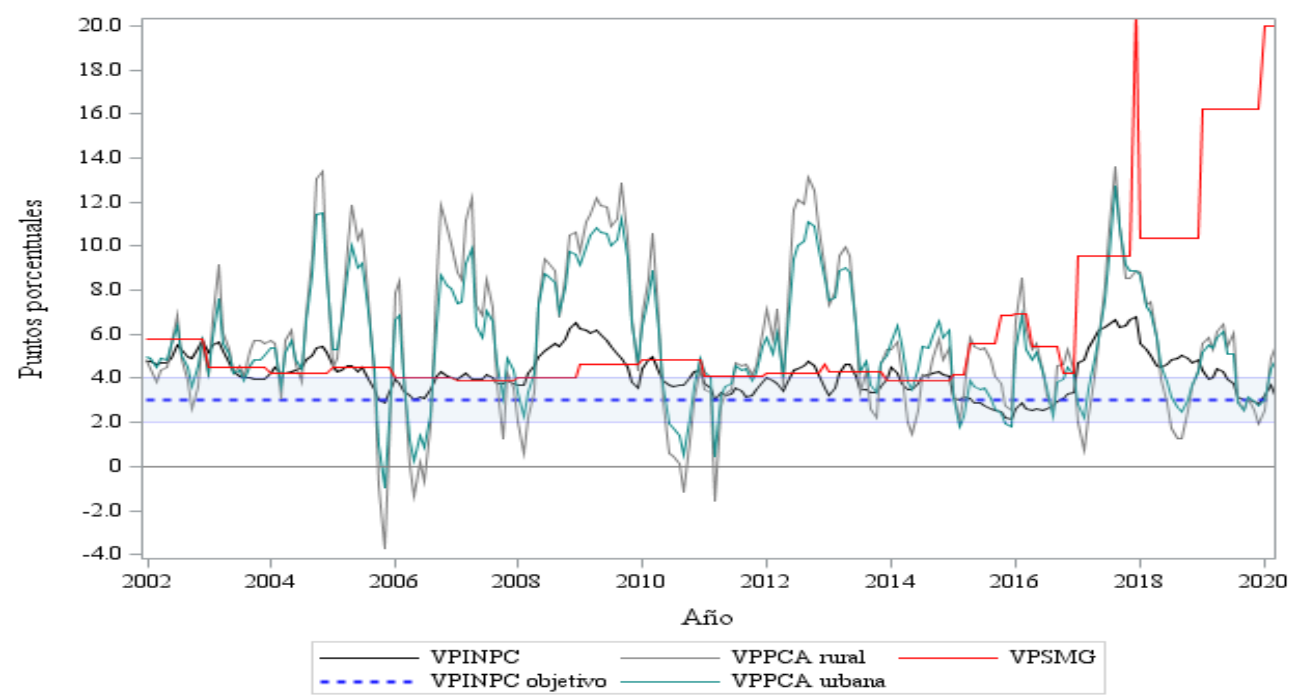

Figura 1. Variación porcentual (VP) del INPC, PCA y SMG nominal, 2002:01-2020:06. La banda color azul es rango de inflación ( $3 \% \pm 1 \%)$ de BANXICO. Con datos de INEGI (2020); CONEVAL (2020); CONASAMI (2020).

En términos reales el SMG ha sufrido un profundo deterioro durante varias décadas al punto que su monto no cumple con las normas de la Constitución (Moreno et al., 2014). Las grandes brechas de separación que ha experimentado México respecto al incremento porcentual en cuanto a los precios, cuando estos han superado a los aumentos salariales, pueden traducirse como una pérdida de poder adquisitivo en los años donde se presentó, impactando en personas más desprotegidas que viven verdaderamente con dicha retribución en cuestiones muy básicas. Asimismo, se reitera la mínima capacidad del SMG que tiene sobre la inflación general, mientras esta sea alta y sobresalga de las bandas de margen de maniobra de BANXICO mismas que deben ser acompañadas de buenas prácticas en política implementadas desde 2001 por este banco central, las cuales han impactado positivamente en control de inflación, principalmente a inicios de 2015 con niveles bajos hasta octubre 2016; no obstante, estas no han sido suficientes para que se vean reflejados en el SMG real.

En 2020 la VPINPC en marzo, abril y mayo ha sido de 3.25, 2.15 y $2.84 \%$ menor que el año pasado; no obstante, ante el panorama de la crisis sanitaria por COVID-19 los patrones de consumo, uso de bienes, servicios y precios de los alimentos principalmente pueden tener nuevos comportamientos (Figura 2).

En periodos posteriores al nivel de inflación más alto (año 2017), se comprueba cómo durante 2018-2020 el SMG nominal se incrementó 39.45\%, mientras el SMG real sólo 31.31\%. Sin embargo, en los dos últimos años 2019 y 2020 el incremento en el SMG nominal ha sido importante, siendo 16.21 y $20 \%$, al igual que en el SMG real 12.13 y $17.11 \%$ respectivamente (Figura 1 y 2). De acuerdo con Díaz (2020), en 2020 el SMG nominal debió haber sido \$320.00 pesos; es decir, más del doble del registrado en este mismo periodo ( $\$ 123.22$ pesos), para compensar el rezago o estancamiento que ha mantenido el SMG real; sin embargo, aumentos de gran magnitud deben ser graduales, para lograr reducir la brecha existente entre ambos. 


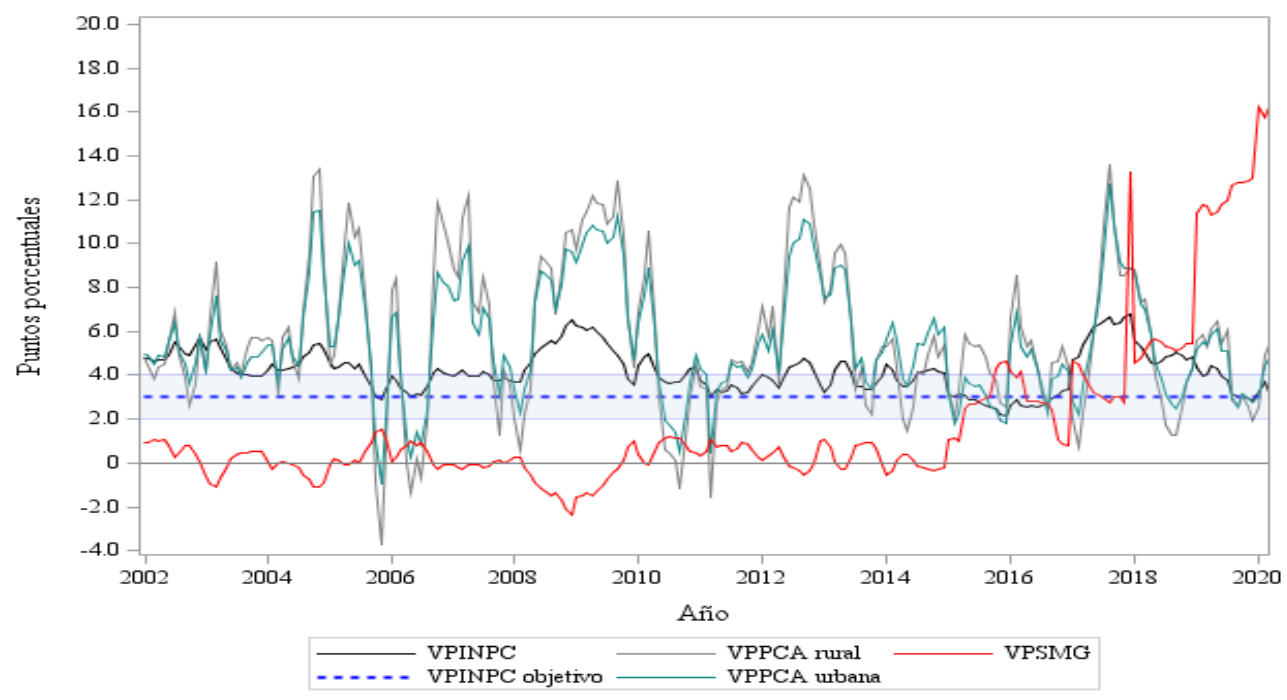

Figura 2. Variación porcentual (VP) del INPC, PCA y SMG real, 2002:01-2020:06. La banda color azul es rango de inflación $(3 \% \pm 1)$ de BANXICO. Con datos de INEGI (2020); CONEVAL (2020); CONASAMI (2020).

De acuerdo con Galicia y Martínez (2020), el comportamiento de la VPINPC durante 2018 se mantuvo fuera del objetivo de Banco de México (BANXICO). Sin embargo, periodos posteriores se ha conservado a la baja, donde la inflación subyacente apenas varió 0.94 puntos porcentuales, en contraste con la inflación no subyacente la cual no omite precios volátiles como los combustibles, entre otras mercancías, al colocarse entre 6.99 y $9.15 \%$.

La inflación asociada a energéticos y tarifas gubernamentales autorizadas, registró un repunte desde el segundo trimestre 2018 extendiéndose hasta septiembre, siendo contrario el comportamiento del componente de productos agropecuarios; sin embargo, a pesar del ritmo creciente observado durante 2018, la VPINPC comenzó a descender a inicios de septiembre 2018, coincidiendo con el desempeño del subíndice no subyacente, específicamente el de energéticos, situación que ha impactado en mejorías a la inflación general objetivo en 2019 en un ritmo más lento asociado al despacio crecimiento económico

No obstante, las tendencias de expectativas mostradas a la baja desde 2018 en la inflación general han sido presionadas por el entorno externo y el cambio de administración gubernamental. En particular, la evolución de los precios de los carburantes debe cuidarse debido a que tienen una alta incidencia en la inflación, agravándose si el tipo de cambio se deteriora, ya que $70 \%$ del consumo nacional de gasolina y diésel proviene del exterior, donde al menos 50\% de la constitución del precio al que se venden en el mercado se explica por el pago de los distribuidores a proveedor (Galicia y Martínez, 2020).

Un aspecto clave durante el sexenio 2013-2018, en el programa sectorial de desarrollo social, anunció que, entre los causantes principales de los elevados niveles de pobreza de la población, se encuentra crecimiento de los precios de los alimentos que ha repercutido en el poder adquisitivo del ingreso de las familias (SEDESOL, 2018), siendo la principal explicación al desconcertado comportamiento de los indicadores de pobreza por ingresos (Noriega, 2019). De esta manera, 
Noriega (2019) asume que al recuperarse la producción de alimentos principalmente en Estados Unidos de América (EE. UU) y reducirse los precios de cultivos básicos en el mundo, desciende un poco la inflación, cifra que representa menos de la mitad de los años anteriores.

El comportamiento de los precios en México de los energéticos, frutas y verduras, otros servicios, alimentos procesados y productos pecuarios, marcan principalmente el ritmo de ascenso o descenso en el INPC, en la composición de dicho índice los productos agrícolas representan $5.1 \%$, productos cómo el jitomate, papa y otros tubérculos, frijol y manzana contribuyen con la mayor participación, mientras que los pecuarios $6.6 \%$, siendo la carne de res, pollo, cerdo y huevo los que aportan la mayor parte (SIAP, 2020).

Sin embargo, se observa que una verdadera política de precios no se ha implementado, habiendo repercusiones de subidas de precios principalmente en el sector agropecuario que es donde verdaderamente están productos afectados de estacionalidad e incertidumbre climática, impactando en hábitos de consumo de los individuos y de familias, quienes apenas alcanzan para comprar productos necesarios que doten de energía necesaria al cuerpo.

Según Noriega (2019), el nivel de ingreso de más de $20 \%$ de la población nacional esta compactado, al destinar la mayor proporción a alimentos, viéndose afectados por el aumento escalonado de precios, dicho comentario lo concreta con lo estipulado por SEDESOL (2018) en el programa sectorial de desarrollo social, al mencionar que la mayor parte del periodo 2005-2013, la tasa de crecimiento del valor de las canastas alimentarias fue mayor que la inflación promedio, traduciéndose en un menor poder de compra de los alimentos, con efectos particularmente a las familias de menores ingresos, las cuales destinan a la compra de alimentos una mayor proporción de su gasto total.

El artículo 123 de la Constitución de los Estados Unidos Mexicanos prescribe 'los salarios mínimos generales deberán ser suficientes para satisfacer las necesidades normales de un jefe de familia, en el orden material, social y cultural y para proveer a la educación obligatoria de los hijos' (Guerrero y Lomelí, 2017). Sin embargo, en el citado artículo se refiere a un individuo por familia, siendo así, dicho salario ha sido insuficiente para dos personas.

Esfuerzos por parte de CONEVAL para medir la pobreza, en base a la evolución del valor de la CA arrojan información valiosa para estar monitoreando líneas de pobreza precisamente por ingresos, por parte de la población y conocer precisamente su comportamiento, ya que CONEVAL define a la línea de pobreza extrema por ingreso a quienes solo pueden o están en potencia de adquirir la CA, mientras que la línea de pobreza por ingresos son los que pueden o están en potencia de adquirir la CA ampliada (canasta alimentaria más no alimentaria), siendo los primeros más vulnerables y quienes se debe implementar una política de compensación salarial que ayude a su ingreso y sus necesidades básicas, acompañado de programas y acciones de desarrollo social.

De acuerdo a Heath (2012), en los últimos años el incremento porcentual de la CA, es superior a la inflación, que hasta el momento se usa para determinar incrementos salariales. México enfrenta la contingencia sanitaria en condiciones de vulnerabilidad, la crisis humanitaria por COVID-19 pone en riesgo avances en desarrollo social y puede afectar en mayor magnitud a grupos vulnerables, la pobreza por ingresos podría ascender entre 7.2 y $7.9 \%$, teniendo un aumento de la pobreza extrema por ingresos entre 6.1 y 10.7 millones de personas para 2020 (CONEVAL, 2020). 
Actualmente ante el panorama mundial se busca evitar que la crisis sanitaria del COVID-19 no se transforme en una crisis alimentaria, donde los canales de transmisión de los impactos de la pandemia son: la demanda, oferta y comercio internacional; asimismo, se reitera probablemente cambios en los patrones de ingreso y consumo, en estas circunstancias se agravaría la desigualdad que precedía a la pandemia, ya que hogares de menores ingresos consumen más granos, panes y cereales y menos lácteos, carnes y huevos que los hogares de mayores ingresos. Los mercados internacionales responden bien, pero su resiliencia no está asegurada si la pandemia se extiende en el tiempo, quienes hasta ahora muestran una situación favorable (CEPAL y FAO, 2020).

\section{Conclusiones}

Se comprobó cómo los precios de la $\mathrm{CA}$, crecen a un ritmo más rápido que el SMG. Esta situación refleja que los individuos se han encontrado en episodios por debajo de la línea de pobreza alimentaria, como el mostrado en el periodo 2002-2020, donde no han estado en condiciones favorables de mejorar su condición económica, mostrando reducida capacidad adquisitiva. Sin embargo, debido a aumentos recurrentes anticipados en SMG nominal, en diciembre 2017 y nuevas reformas en años subsecuentes implementadas por la CONASAMI en 2019 y 2020 se han logrado incrementos en 16.21 y 20\%, dichas acciones ante un nuevo modelo de recuperación gradual y sostenido del SMG, mismo que ha ayudado para que al menos este se encuentre por arriba de la inflación general y de los precios de la CA rural y urbana.

Al investigar periodos posteriores (2017) al nivel de inflación más alto, se demuestra cómo durante 2018-2020 el SMG nominal se incrementó 39.45\%, mientras el SMG real sólo 31.31\%. No obstante, en los dos últimos años 2019 y 2020 el incremento en el SMG nominal ha sido importante, siendo 16.21 y $20 \%$, al igual que en el SMG real 12.13 y $17.11 \%$ individualmente. Sin embargo, para compensar el rezago que ha mantenido este último, el SMG nominal debió rondar más del doble del registrado en 2020 (\$123.22 pesos) para alcanzar a reducir la brecha existente entre ambos, donde los esfuerzos dependerán de la estabilidad en la inflación y que esta no se contraponga con el SMG, para alcanzar a disminuir la gran brecha diferencial existente entre el SMG real.

\section{Literatura citada}

Alonso, J. C. y Rivera, A. F. 2017. Pronosticando la inflación mensual en Colombia un paso hacia delante: una aproximación 'de abajo hacia arriba'. Revista de Métodos Cuantitativos para la Economía y la Empresa. 23:98-118.

Brambila, P. J. 2011. Bioeconomía: instrumentos para su análisis económico. $1^{\text {a }}$ (Ed.). Secretaría de Agricultura, Ganadería, Desarrollo Rural, Pesca y Alimentación (SAGARPA). 313 p.

CONEVAL. 2018. Consejo Nacional de Evaluación de la Política de Desarrollo Social. Metodología para la medición multidimensional de la pobreza en México. Anexo único de los 'lineamientos y criterios generales para la definición, identificación y medición de la pobreza'. $68 \mathrm{p}$.

CONEVAL. 2020. Consejo Nacional de Evaluación de la Política de Desarrollo Social. La política social en el contexto de la COVID-19 en México. 24 p.

CONEVAL. 2020. Consejo Nacional de Evaluación de la Política de Desarrollo Social. Evolución del valor de la canasta alimentaria. Valor de la canasta alimentaria y no alimentaria. https://www.coneval.org.mx/medicion/mp/paginas/lineas-de-bienestar-y-canastabasica.aspx.0. 
CEPAL y FAO. 2020. Comisión Económica para América Latina y el Caribe y Organización de la Naciones Unidas para la Alimentación y la Agricultura. Cómo evitar que la crisis del COVID-19 se transforme en una crisis alimentaria: acciones urgentes contra el hambre en América Latina y el Caribe. 33 p.

CEFP. 2017. Centro de Estudios de las Finanzas Públicas. Evolución de precios. Cámara de Diputados. LXIV Legislatura del Congreso de la Unión de México. 3 p.

CONASAMI. 2020. Comisión Nacional de los Salarios Mínimos. Tabla de salarios mínimos generales y profesionales por áreas geográficas. https://www.gob.mx/conasami/ documentos/tabla-de-salarios-minimos-generales-y-profesionales-por-areas-geograficas.

CONASAMI. 2020. Comisión Nacional de los Salarios Mínimos. Informe mensual del comportamiento de la economía. $131 \mathrm{p}$.

CEDRSSA. 2017. Centro de Estudios para el Desarrollo Rural Sustentable y la Soberanía Alimentaria. Resultados del índice de la tendencia laboral de la pobreza nacional y rural del tercer trimestre de 2017. Cámara de diputados LXIII Legislatura del Congreso de la Unión de México. 18 p.

Díaz, C. M. 2020. El salario real en México 2015-2019. Economía Actual. 13(1):17-20.

Díaz, C. M. 2020. Inflación y salarios reales en México 2015-2019. Economía Actual. 13(2):11-14.

Dornsbusch, R.; Fischer, S. y Startz, R. 2009. Macroeconomía. 10 (Ed.). Mc Graw Hill. 607 p.

Flores, C. L. 2017. Pronóstico del Índice Nacional de Precios al Consumidor. Rev. Iberoamericana de Contaduría, Economía y Administración. 6(12):60-88.

Galicia, M. U. y Martínez, N. A. 2020. Un modelo no monetario de la inflación en México, 20072018. Rev. de Economía y Administración. 38:135-170.

Guerrero, C. y Lomelí, L. 2017. Reflexiones teóricas en torno a la propuesta de recuperación del poder de compra del salario mínimo en México. Contaduría y Administración. 62(3):958971.

Heath, J. 2012. Lo que indican los indicadores: cómo utilizar la información estadística para entender la realidad económica de México. INEGI. 415 p.

INEGI. 2018. Instituto Nacional de Estadística y Geografía. Índice Nacional de Precios al Consumidor (INPC). Documento metodológico. Base $2^{\text {da }}$ quincena de julio 2018. $137 \mathrm{p}$.

INEGI. 2020. Instituto Nacional de Estadística y Geografía. Índice Nacional de Precios al Consumidor. https://www.inegi.org.mx/temas/inpc/.

INEGI. 2020. Instituto Nacional de Estadística y Geografía. Calculadora de inflación. https://www.inegi.org.mx/app/indicesdeprecios/documentos/Calculadora_de_ Inflacion.pdf.

Moreno, B. J.; Garry, S. y Monroy, G. F. 2014 El salario mínimo en México. EconomíaUniversidad Nacional Autónoma de México (UNAM). 11(39):78-93.

Noriega, A. E. 2019. La inflación alimentaria en 2018. Centro de estudios para el desarrollo rural sustentable y la soberanía alimentaria (CEDRSSA). Dirección de estudios sobre la soberanía alimentaria y nueva ruralidad. Cámara de diputados LXIII Legislatura. México. 23 p.

SIAP. 2020. Servicio de información Agroalimentaria y Pesquera. Secretaría de Agricultura y Desarrollo Rural (SADER). Índice Nacional de Precios al Consumidor (INPC). 4 p.

SEDESOL. 2018. Secretaría de Desarrollo Social. Programa Sectorial de Desarrollo Social 20132018. México. 100 p. 